\title{
Prospect of Polypill in the Management of Cardiovascular Diseases in Bangladesh
}

\author{
Nushrat Alam, Md. Elias-Al-Mamun ${ }^{2}$ and Muhammad Rashedul Islam² \\ ${ }^{1}$ Department of Pharmacy, Faculty of Pharmacy, University of Dhaka, Dhaka-1000, Bangladesh \\ ${ }^{2}$ Department of Pharmaceutical Technology, Faculty of Pharmacy, University of Dhaka \\ Dhaka-1000, Bangladesh
}

(Received: December 27, 2019; Accepted: January 28, 2020; Published: January 30, 2020)

\begin{abstract}
Cardiovascular disease (CVD) is one of the most prominent health issues responsible for global death currently. Just like the diverse causes of its pathophysiology, a plethora of medicines from different classes are used for the treatment and management of CVDs. However, in low and middle income countries like Bangladesh, it's extremely difficult for patients to adhere to their treatment regimen consisting of multiple drugs from different classes as it is both expensive and inconvenient. In such scenario, a polypill or a combination of drugs containing different type of cardiovascular drugs could significantly improve the management of cardiovascular diseases in Bangladesh. Our aim was to get a real-life perspective of the prospect of commercially available polypill in Bangladesh keeping all its stakeholders such as patients, physicians and retailers in mind. A survey was conducted in two different cities (Dhaka and Comilla) and the data were correlated. We got positive response from patients, physicians and retailers. Also, the physicians came up with various combinations of drugs such as ACEI (ngiotensin-converting enzyme inhibitors) and ARB (angiotensin II receptor blockers) (35\%); ARB and CCB (calcium channel blockers) (25\%) and ACEI and CCB (15\%). However, disease patterns, available drugs and comorbidities in the two regions showed significant deviations as metro city based patients suffered from more severe form of CVDs. Therefore, further survey in different regions should be conducted to understand the overall disease pattern and to come up with optimum combinations for CVD patients. Beyond this discussion, it was evident that commercially available polypill has a great prospect in Bangladesh against CVDs.
\end{abstract}

Keywords: Cardiovascular diseases, polypill, ACEIs, ARBs, beta blockers, calcium channel blockers.

\section{Introduction}

Cardiovascular disease is a group of conditions that affects the functioning of the heart or blood vessels. At present, it is one of the leading causes of global death and is responsible for $75 \%$ of the deaths occurring in developing countries, including Bangladesh. CVD prevalence is also on the rise among the adults in Bangladesh due to the rapid urbanization and fast economic growth, habituation of a sedentary life style, changing food habits including growing access to and demands for processed food, inconsistent meal times and reduced physical activity (Misra et al., 2007). Besides, ethnicity, smoking and smokeless tobacco use, dyslipidaemia, life-style related factors, chronic arsenicosis, air pollution also make its people more susceptible to cardiovascular diseases (Islam et al., 2017). The possible treatment options for the management of CVDs are lipid lowering agents, vasodilators, beta blockers, ACE inhibitors, ARBs, Ca channel blockers, diuretics etc. In 2012, a study was conducted in National Institute of Cardiovascular Diseases (NICVD), Dhaka to understand the prescription pattern at a tertiary level hospital in

Correspondence to: Md. Elias Al-Mamun; E-mail: elias_0276@yahoo.com

DOI: https://doi.org/10.3329/bpj.v23i1.45315 
Bangladesh. It revealed that on an average, the patients were prescribed 6.35 drugs (Muhit et al., 2012). Undoubtedly, this is a major problem for chronic CVD patients to adhere to their treatment regimen.

A polypill is a medicine that has different drugs in one pill to minimize the chances of a patient missing out on his/her medications. Apart from improved efficacy, a combination of fixed doses of drugs may have additional advantages such as simplicity in use, scalability and improved patient adherence. Wald and Law first proposed a fixed dose combination (FDC) consisting of half-therapeutic doses of statin, two antihypertensive agents, aspirin, and folate (Wald and Law, 2003) and endorsed the idea of prescribing 'polypill' to everyone over the age of 55 without screening. But this idea further gave rise to several other questions. For example, what should be the doses of individual drugs in a FDC? Besides, a large number of cardiovascular patients suffer from additional kidney diseases, diabetes and other problems which should be taken into consideration before choosing the optimum combination for the management of CVD patients which raised the most crucial question for the commercialization of a polypill - would a single cardiovascular FDC achieve its desired adherence in patients with multiple pathologies such as diabetes, osteoarthritis, and psychological illness? Or a wider range of FDCs need to be developed for multiple common disease combination rather than cardiovascular diseases alone? The use of drugs for the treatment of diabetes and other pathologies may itself increase the risk from cardiovascular complications due to their direct effect on the drug's molecular actions or indirect effect that might result from changes in the metabolic profile of the patient. So, if we consider to launch polypill in the market for the management of cardiovascular diseases, at first we have to gather information on the disease pattern, current approach of treatment, co-morbidities associated with CVDs and more importantly whether our patients will be keen on taking one pill instead of multiple pills or are there any objection from physicians and retailers as they play crucial role in the chain of disease management. That's why we tried to find out the real scenario of polypill whether these combinations will be beneficial for the patients or not.

\section{Materials and Method}

Design of experiment: We reviewed necessary literature and existing similar system in different countries. Depending on gathered data from literature review we had made our plan to carry out the research. The next step for this project was data collection. In this step, we studied current research and clinical trials regarding use of combination pills. Then we conducted a survey to identify the current treatment approach for CVDs to the doctors, the prospects of the possible polypill and the opinion of doctors, patients and retailers regarding commercially available polypill.

Selection of stakeholders: The respondents of this study were the doctors, patients and retailers. We selected National Institute of Cardiovascular Disease (NICVD), Dhaka and Comilla Medical College and Hospitals for interviewing patients and doctors. Retailers from Dhaka city were also chosen.

Data sources: To carry out the study, sources of both primary and secondary data were used. The main respondents under this study were the doctors, patients and retailers. Secondary information was collected from the various reports, research journals, newspapers, related books, articles and online sources.

Data collection: Required information about the study area was attained by preliminary field investigation. Respondent's perceptions and suggestions were taken from the selected medical facilities to fulfill specific purpose of the research within the particular time. To find the prospect of polypill in the treatment and management of cardiovascular diseases in Bangladesh, sample survey was conducted in different medical colleges among 18 doctors, 24 patients and 14 retailers. Primary data was collected using the interviews of the respondents from selected medical colleges of the study area. The methods of data collection were survey method 
(Figure 1) by using different structured questionnaire to different stakeholders.
Data analysis: Findings from our studies were processed and analyzed by using both descriptive and inferential statistical data by using MS Excel.

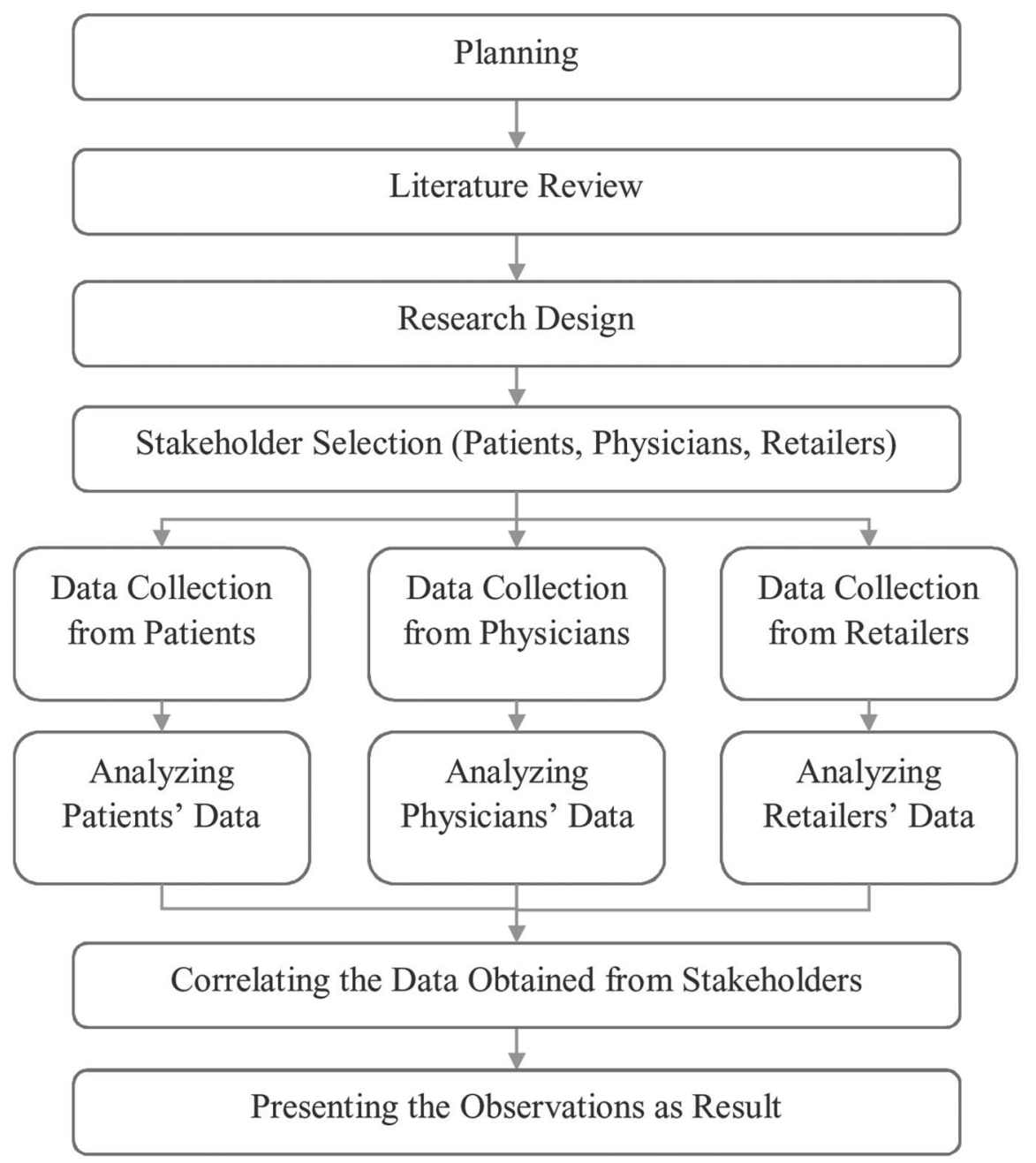

Figure 1. Methodology flowchart.

\section{Results}

Current scenario of cardiovascular diseases in Bangladesh: 94.44\% Physicians agreed that CVD is an emerging health issue in Bangladesh while 5.56\% physicians concluded that the number of patients suffering from CVDs has been consistent. Based on the data collected, $58.33 \%$ patients were suffering from chest pain, $20.83 \%$ were suffering from asthma, $4.17 \%$ patients had hyperlipidemia and $12.5 \%$ complained of partial heart blockage who had also undergone surgery (Figure 2). Among the patients, $70.83 \%$ respondents were also suffering from chronic hypertension. However, the scenario was different in the two cities as in Dhaka alone, $85.71 \%$ patients were suffering from hypertension (Figure 3) while the percentage in Comilla was 50\% (Figure 4).

Susceptibility to cardiovascular diseases: According to the physicians, $59.25 \%$ of total CVD patients belonged to the age group of 40 or more years. On the other hand, $22.22 \%$ patients belonged to the age group of 30-40 years, while the remaining $18.52 \%$ patients belonged to the age group of 20-30 years. Based on the data collected from retailers, $93.33 \%$ of the customers were 40 years old or older 
while $6.63 \%$ were aged between 30 and 40 years. Individually smoking, family history of HTN, stress and obesity were blamed for the development of CVDS. However, the physicians pointed out that two third of the patients had all these risk factors. The remaining $33.33 \%$ patients were smoker with one or more risk factors.

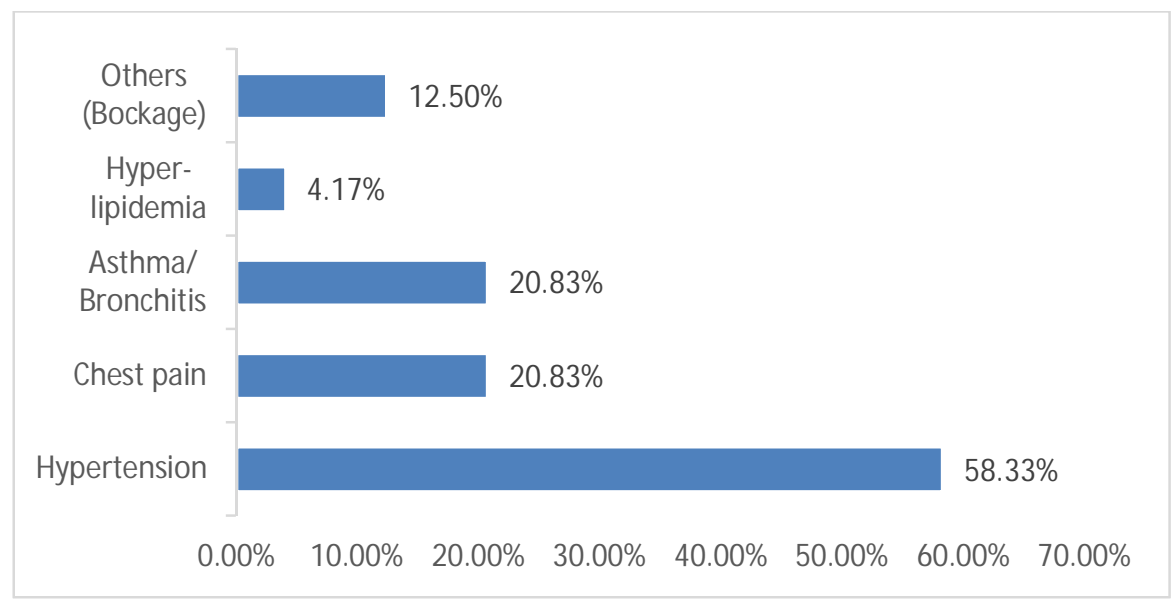

Figure 2. Types of CVDs patients suffer from.

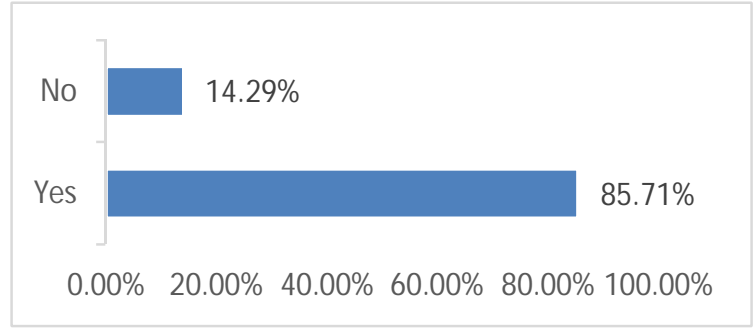

Figure 3. Hypertension (HTN) scenario in Dhaka.

Correlation of CVDs with diabetes and/or chronic kidney diseases (CKD): $45.83 \%$ Patients complained of having diabetes and/or chronic kidney disease, whereas $54.17 \%$ patients had no history of either of the diseases. However, this situation varied markedly in Dhaka and Comilla. In Dhaka alone, $57.14 \%$ patients were diagnosed with diabetes and/or CKD while the percentage in Comilla was only $30 \%$. We obtained similar data from the physicians. While they pointed out various complications such as stroke (30.56\%), DM (Diabetes mellitus) (11.11\%) and CKD (11.11\%) associated with chronic hypertension (Figure 5) and the issue was more prominent in Dhaka than Comilla (Figures 6, 7). In fact, in Dhaka, patients with chronic hypertension were at risk of

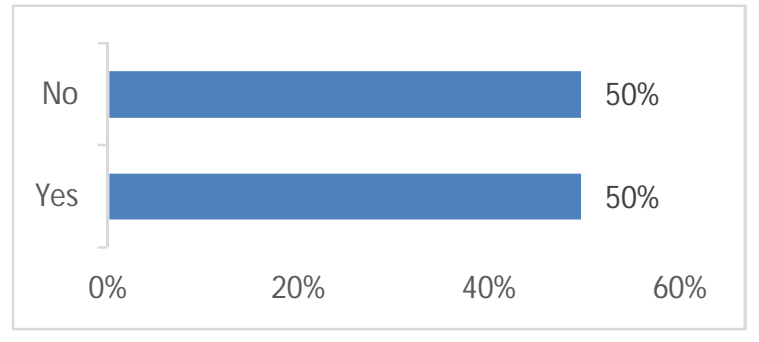

Figure 4. Hypertension (HTN) scenario in Comilla.

developing DM and CKD more than any other cardiovascular diseases.

Number of medicines taken by CVD patients on daily basis: Among the patients, $45.83 \%$ usually take one medicine for CVD on a daily basis, $29.17 \%$ take two medicines, $8.33 \%$ take three medicines and the remaining (16.67\%) patients take four or more medicines. According to the retailers, $100 \%$ patients who were suffering from CVDs bought more than one drug at a time.

Patient adherence: $54.17 \%$ Patients told they were adherent to their daily dose of medicine. Remaining $45.83 \%$ admitted that sometimes they skipped their medication. This situation was more alarming in Comilla as $90 \%$ of patients skipped their medicine at times while the percentage was only 
$28.57 \%$ in Dhaka. Similarly, according to $44.44 \%$ physicians, patients took multiple medicines satisfactorily while $55.56 \%$ responded in negative.

Response to polypill: About $79.17 \%$ patients were positive about launching a polypill, whereas the remaining $20.83 \%$ respondents were satisfied taking multiple pills individually. In case of physicians, $83.33 \%$ doctors told they thought polypill would be a better option for the management of CDVs while $11.11 \%$ doctors were not sure about the outcome.
The remaining $5.56 \%$ doctors preferred individual medicine for patients. While we interviewed retailers, $78.57 \%$ retailers concluded that a polypill would be commercially successful in our market. $41.67 \%$ retailers stressed on cost effectiveness, $8.33 \%$ on therapeutic effectiveness, $8.33 \%$ on patient adherence while the remaining $41.67 \%$ pointed out all these benefits.

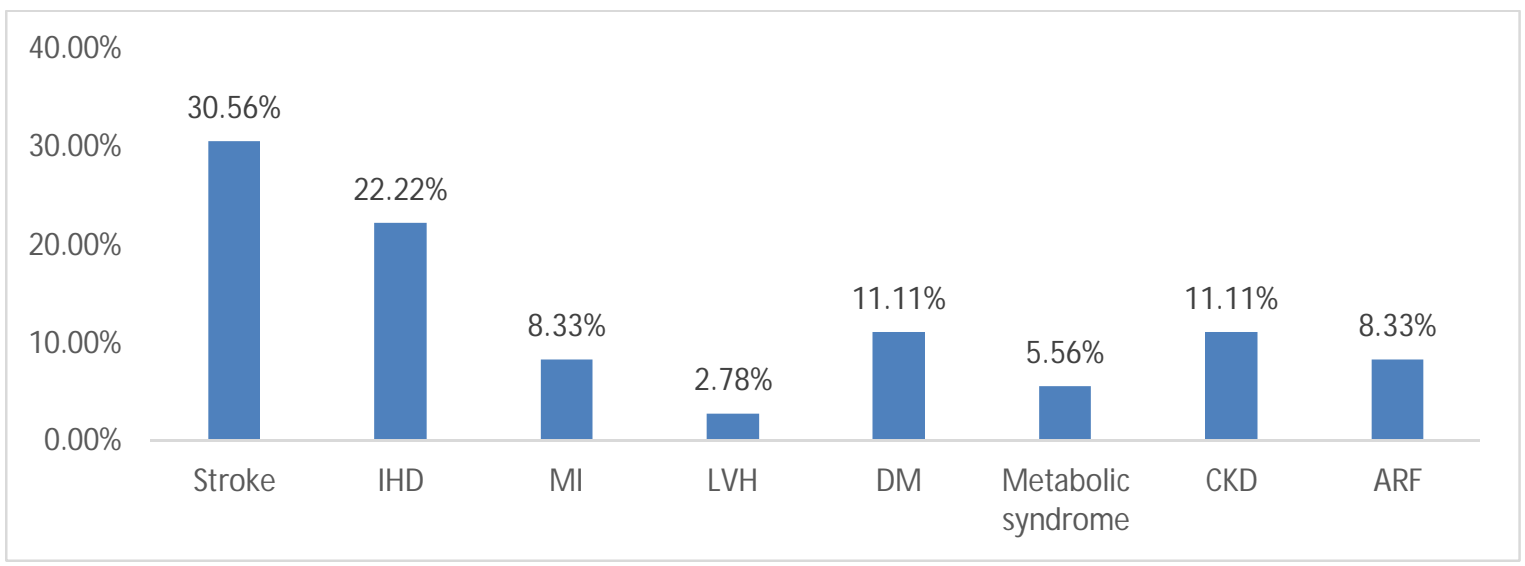

Figure 5. Additional complications associated with cardiovascular patients.

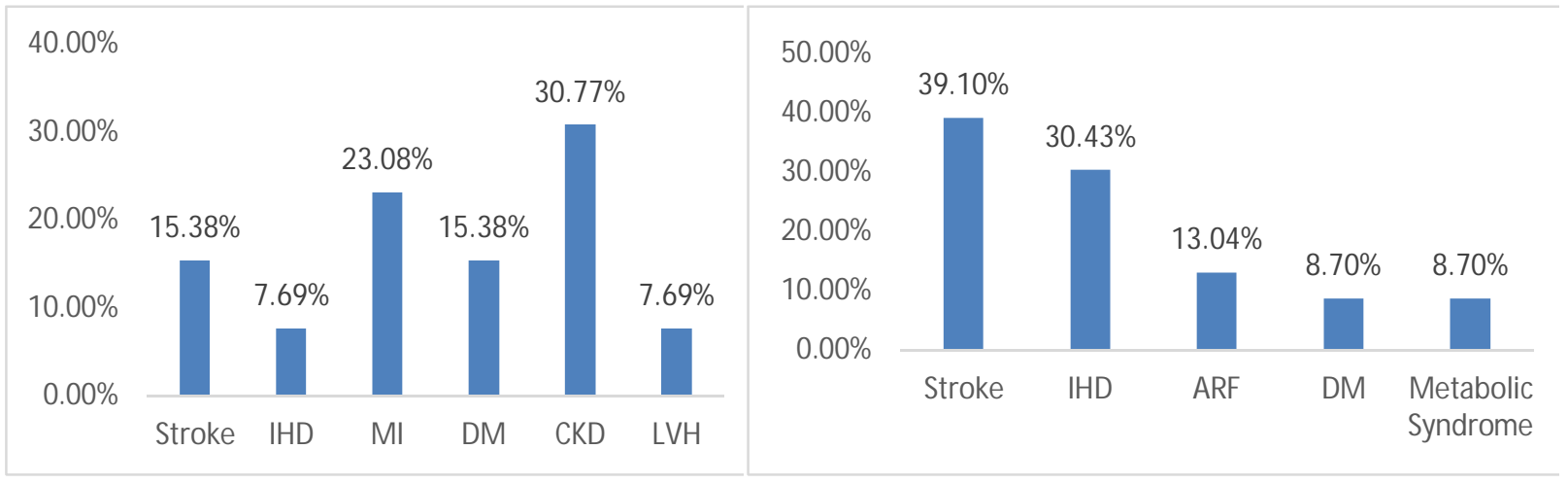

Figure 6. Additional complications in Dhaka.

Figure 7. Additional complications in Comilla.

Probable combination of drugs: According to CVD patients and retailers, ARBs (20.41\%), antiplatelet drugs (39.02\%), anti-asthmatic drugs, beta blockers, anti-anginal drugs and combined antihypertensive drugs were the major classes of drugs used in the management of CVDs (Figures 89).
On asking which combination of drugs would be better for managing cardiovascular patients, $10 \%$ physicians opted for a combination of beta blocker and $\mathrm{Ca}$ channel blocker, $5 \%$ physician wanted to use a combination of ACEI, beta blocker and Ca channel blocker, $35 \%$ physicians preferred a combination of ACEI and beta blocker, $15 \%$ physician wanted to use 
a combination of ACEI and Ca channel blocker, 25\% physician wanted to use a combination of ARB and Ca channel blocker and 5\% physician wanted to use a combination of ARB and diuretics. Finally, 5\% physicians preferred to use a combination of antiplatelet drugs, lipid lowering agents and beta blockers (Figure 10).
However in Dhaka region, the most preferred combination was that of $\mathrm{ARB}$ and $\mathrm{CCB}$ which constituted of $50 \%$ of total share (Figure 11). In Comilla region, the most preferred combination was ACEI with beta blocker constituting $60 \%$ of the total share (Figure 12).

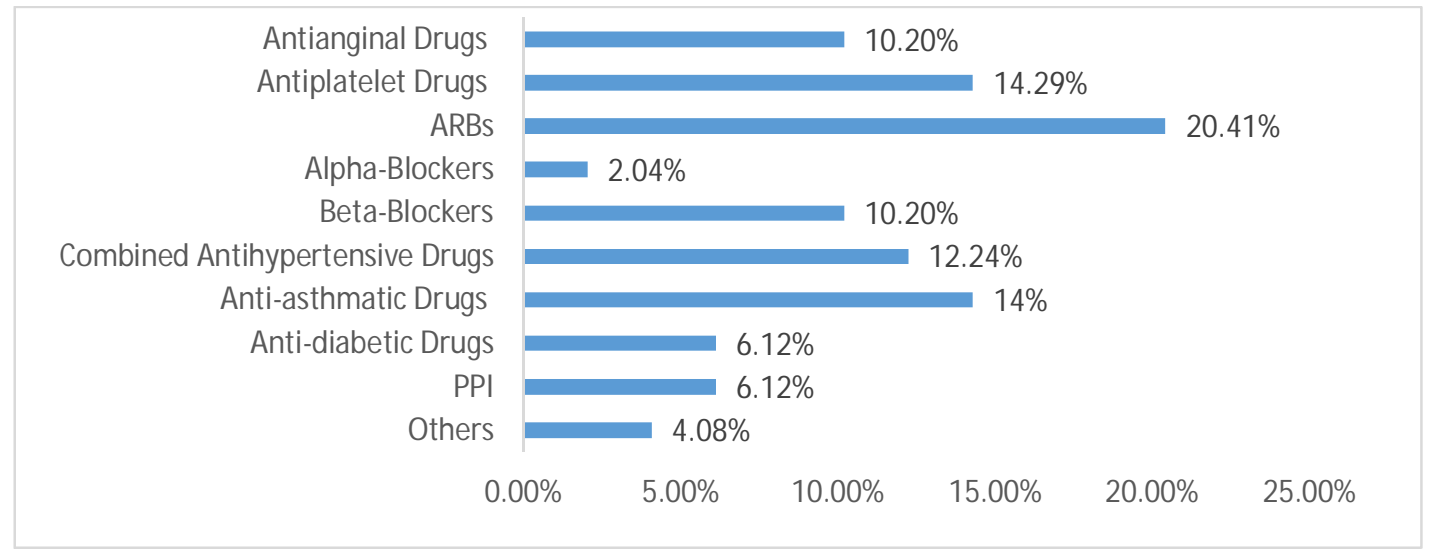

Figure 8. Data obtained from patients.

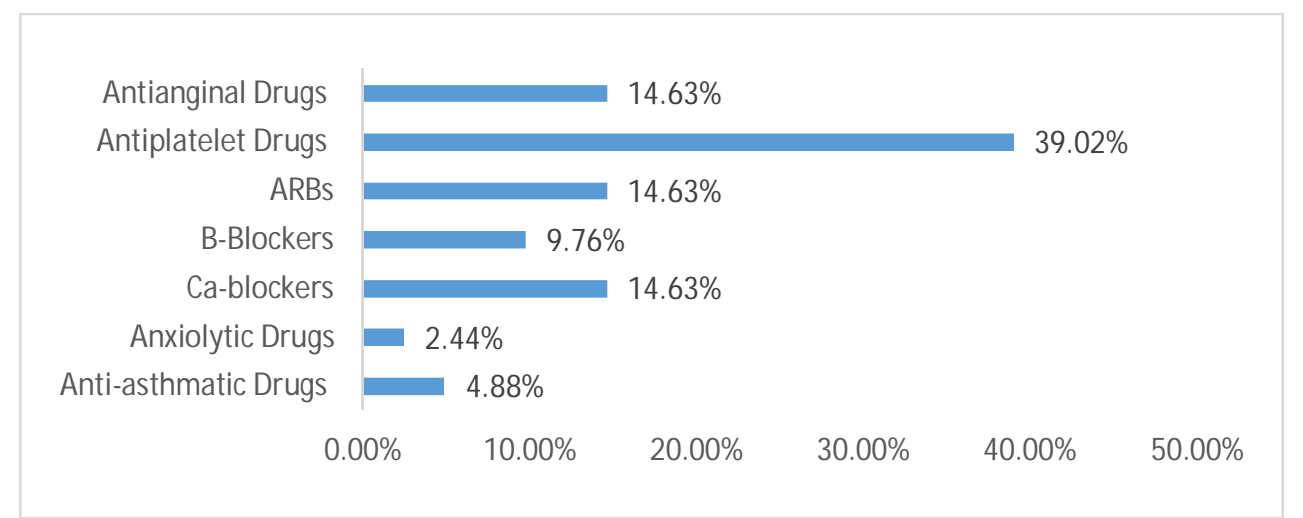

Figure 9. Data obtained from retailers.

\begin{tabular}{|c|c|c|c|c|}
\hline B blocker +Ca Blocker & 10. & & & \\
\hline ACEI +B Blocker & & & & $35.00 \%$ \\
\hline ACEI + Ca Blocker & 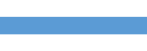 & $5.00 \%$ & & \\
\hline ACEl + Beta blocker + Ca Blocker & $5.00 \%$ & & & \\
\hline ARB + Ca Blocker & & & $5.00 \%$ & \\
\hline ARB + Diuretics & $5.00 \%$ & & & \\
\hline Antiplatelet Drugs + Lipid Lowering Agents $+B$ blockers & $5.00 \%$ & & & \\
\hline & $10.00 \%$ & $20.00 \%$ & $30.00 \%$ & $40.00 \%$ \\
\hline
\end{tabular}

Figure 10. Combinations of drugs preferred by doctors. 


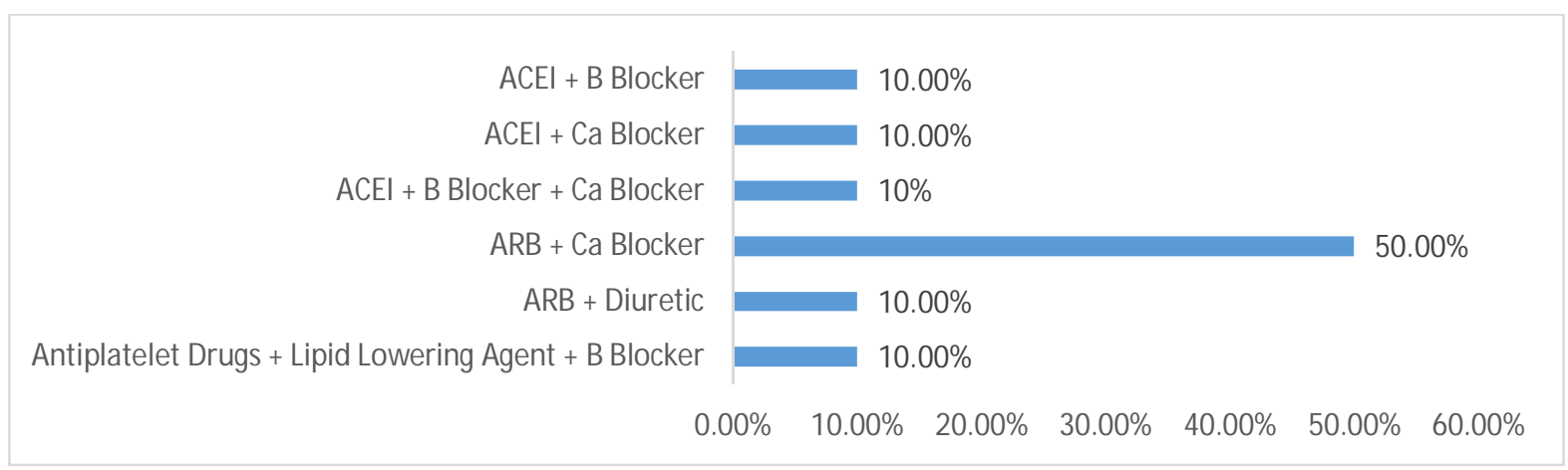

Figure 11. Combinations of drugs preferred by doctors in Dhaka.

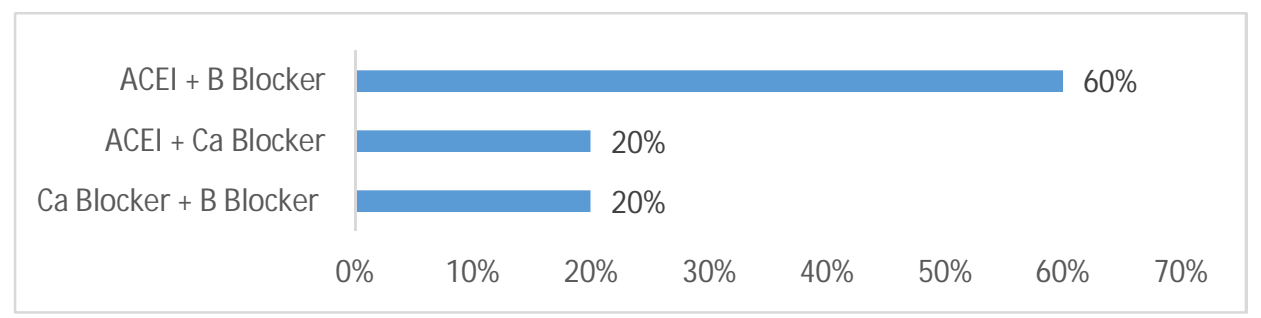

Figure 12. Combinations of drugs preferred by doctors in Comilla.

\section{Discussion}

This study titled prospects of polypill in the management of cardiovascular diseases in Bangladesh was conducted among patients, physicians and retailers. It enabled us to understand the current approach of physicians in treating CVDs, real life implications of a polypill, patient's approach toward it and the possibility of a successful, commercially available polypill in Bangladesh.

The majority of the cardiovascular patients were diagnosed with hypertension. However, we observed that people living in Dhaka had much higher rate of hypertension than those living in Comilla (Figures 34). The next significant portion of patients were suffering from coronary artery disease and hyperlipidemia.These findings were consistent with the fact that due to ethnicity, Bangladeshi people had a high prevalence of CAD (coronary artery disease) and associated risk factors (Yusuf et al., 2001; Palaniappan et al., 2010) and a 3 to 5-fold increased risk of myocardial infarction (Gupta et al., 2006). The respondent physicians also stressed on diabetes and acute and chronic kidney diseases. But there was a significant difference in the data obtained from two cities. While, Dhaka based physicians pointed out stroke, MI and IHD as major cardiovascular complications, they also revealed that majority of their patients suffer from additional problems such as diabetes and chronic kidney diseases which is even more prevalent than the CVDs themselves. However, Comilla based doctors put more emphasis on stroke and IHD (ischemic heart disease) and comparatively lesser number of their patients were suffering from ARF (acute renal failure) or diabetes (Figures 6-7). This observation pointed out towards socioeconomic transition and the resulting change in our lifestyle as well as the dietary pattern. Nowadays people are getting more used to fried foods and in a metro city people hardly get any time or scope for physical exercise. Animal protein-rich diet in rural areas of Bangladesh was associated with increased risk of CVD mortality, especially among smokers (Chen et al., 2013). Besides obesity and abdominal adiposity were associated with development of CAD in Bangladeshi population (Khan et al., 2015). This is consistent with a study published in the June 2012 issue of the journal "Archives of Iranian Medicine" that there has been an increase of CVD in young and 
middle-aged fast-food eaters as eating too much of any of fast foods can alter metabolic factors that increase one's risk for heart disease. We saw a similar trend in our country as our respondent doctors informed us that in recent years, there has been an increase in the number of younger people (20-40 years) suffering from CVDs. Our data obtained from patients and physicians also showed that metro city based patients (people in Dhaka) were suffering from more severe form of CVDs and the tampering of food made them more susceptible to kidney diseases compared to people living in a comparatively less modern city (people in Comilla).

Another unfortunate but important finding was that patients adherence to their treatment was not satisfactory. While almost one third of the patient in Dhaka skipped their medicines at times, it was more alarming in case of Comilla as in $90 \%$ of cases patients failed to take medicines. It pointed to the fact that, in the capital city, education and awareness had a much stronger influence on patients' behavior. While in other part of the country there was a significant breach in protocol. We realized that the major reason behind their skipping medicine is that they don't want to take multiple medicine and whenever they felt okay, they skipped their medicines. It also showed that there is a huge gap in patients' understanding of the gravity of their diseases and they often fail to understand how this discontinuity can affect their health in the long run. So, when the prospective of a polypill came, majority of the respondents were keen on taking one medicine at once instead of taking multiple medicines. The study conducted among physicians and retailers also gave similar results. Physicians were very much open to the idea of the commercially available polypills. However, they wanted to use it in conjunction with adjunct of healthy life style and were concerned with the price. Our data obtained from retailers showed that currently available combination of drugs (combined anxyolytics, combined antihypertensive drugs, combination of aspirin and clopidogrel) are already being well received and preferred by consumers. So when they were asked about their opinion, majority of the retailers were positive about the success of commercially available polypills as long as they were cost effective and don't have additional side effects.

On the question of what should be the primary constituent of polypill(s), physicians came up with multiple possible combinations such as - a combination of beta blocker and $\mathrm{Ca}$ channel blocker, a combination of ACEI, beta blocker and Ca channel blocker, a combination of ACEI and beta blocker, a combination of ACEI and $\mathrm{Ca}$ channel blocker, a combination of $\mathrm{ARB}$ and $\mathrm{Ca}$ channel blocker, a combination of $\mathrm{ARB}$ and diuretics and a combination of antiplatelet drug, lipid lowering agent and beta blocker. However, these data were directed by treatment pattern in a particular region. We observed, in Dhaka, multiple classes of drugs were available to treat CVDs. So, physicians in this region came up with more combinations. While, in Comilla, physicians had limited choice of drugs (ACEI, ARB and beta blockers) and they came up with less varied combinations. Besides, due to the disease pattern, Dhaka based physicians put more emphasize on additional kidney diseases. So, majority of them preferred a combination of $\mathrm{ARB}$ and $\mathrm{Ca}$ channel blocker as both the drugs have additional positive effect on chronic kidney patients and diabetes patients (Figure 11), whereas the doctors in Comilla preferred a combination of ACEI and beta blocker more (Figure 12).

Outside the professional opinion, from the data obtained from retailers, we observed among all classes of cardiovascular drugs, the most distributed drugs are anti-platelets (Figure 9). Considering the risk of IHD, MI and stroke, anti-platelet drugs can be a component when treating patients with severe cardiovascular diseases. Besides, alpha 1-blockers, ACE inhibitors, and calcium channel blockers are well tolerated with patients with hyperlipidemias and sometimes they improve lipid profile (Kannel and Carter, 1989). So, even though alpha 1-blockers are not popular compared to other therapeutic agents, for particularly treating hyperlipidemia patients, we can combine this drug with other drugs. Besides, considering the number of CVD patients with 
diabetes, we can also include such drugs in a combination for its additional cardiovascular benefit. For example - metformin has been found to improve lipoprotein metabolism and a direct/indirect role in the protection of endothelial vascular function.

However, in real life scenario, we cannot simply add different classes of drugs considering their cardio-protective effect. As, such combinations can be harmful for patients with specific disease. Some anti-diabetic drugs have been found to change the metabolic profile of the patient and increase the risk of cardiovascular complications. Therefore, we should begin with an already established combination which has been proved to be efficacious (for example - Ca channel blockers are effective in reducing blood pressure in patients with CKD and work well in a regimen using a diuretic and ARB) and gradually increase the primary component(s) for treating the patients.

\section{Conclusion}

Based on our survey on patients, physicians and retailers, we found that the idea of polypill for the treatment and management of cardiovascular diseases in Bangladesh has a great prospect. The major advantageous point has been the positive outlook of all stakeholders regarding its commercialization. However, we had noticed some major drawbacks too, as treatment patterns as well as the occurrence of additional diseases has been different in two different areas. Thus, it will be difficult to come up with an optimum formulation for a single polypill. Further surveys should be carried out in other major cities, so that we could have a better understanding of the disease pattern and come up with the most optimum formulations of polypills which would be beneficial for CVD patients without any deterioration on other physiologic systems. Finally, we could conclude that polypill has better aspect in the treatment and management of cardiovascular diseases in Bangladesh based on our results.

\section{References}

Chen, Y., McClintock, T.R., Segers, S., Parvez, F., Islam, T., Ahmed, A., Rakibuz-Zaman, M., Hasan, R., Sarwar, G. and Ahsan, H. 2013. Prospective investigation of major dietary patterns and risk of cardiovascular mortality in Bangladesh. Int. J. Cardiol. 167, 1495-1501.

Gupta, M., Singh, N. and Verma, S. 2006. South Asians and cardiovascular risk. Circ. 113, 924-929.

Islam, A.K.M.M., Mohibullah, A.K.M. and Paul, T. 2017. Cardiovascular disease in Bangladesh: a review. Bangladesh Heart J. 31, 80-99.

Kannel, W.B. and Carter, B.L. 1989. Initial drug therapy for hypertensive patients with hyperlipidemia. Am. Heart J. 118, 1012-1021.

Khan, R.J., Harvey, D.J., Leistikow, B.N., Haque, K.S. and Stewart, C.P. 2015. Relationship between obesity and coronary heart disease among urban Bangladeshi men and women. Integr. Obes. Diabetes 1, 49-55.

Misra, A., Misra, R., Wijesuriya, M. and Banerjee, D. 2007. The metabolic syndrome in South Asians: continuing escalation \& possible solutions. Indian $J$. Med. Res. 125, 345-354.

Muhit, M.A., Rahman, M.O., Raihan, S.Z., Asaduzzaman, M., Akbar, M.A., Sharmin, N. and Faroque, A.B.M.F. 2012. Cardiovascular disease prevalence and prescription patterns at a tertiary level hospital in Bangladesh. J. Appl. Pharm. Sci. 2, 80-84.

Palaniappan, L.P., Araneta, M.R., Assimes, T.L., BarrettConnor, E.L., Carnethon, M.R., Criqui, M.H., Fung, G.L., Narayan, K.M., Patel, H., Taylor-Piliae, R.E., Wilson, P.W. and Wong, N.D. 2010. Call to action: cardiovascular disease in Asian Americans. Circ. 122, 1242-1252.

Wald, N. and Law, M. 2003. A strategy to reduce cardiovascular disease by more than $80 \%$. British Med. J. 326, 1419-1424.

Yusuf, S., Reddy, S., Ôunpuu, S. and Anand, S. 2001. Global burden of cardiovascular diseases: part II: variations in cardiovascular disease by specific ethnic groups and geographic regions and prevention strategies. Circ. 104, 2855-2864. 